\title{
Characterising the microstructure of random media using ultrasound $\dagger$
}

\author{
M F Insana and T J Hall \\ Department of Diagnostic Radiology, University of Kansas Medical Center, Kansas City, \\ KS 66103, USA
}

Received 6 March 1990, in final form 10 May 1990

\begin{abstract}
This paper describes a technique of forming images of the average size and scattering strength of scatterers in a random medium using ultrasound. Quantitative ultrasound images provide a more direct interpretation of the underlying structure of the medium, e.g. size, shape, number and elastic properties of scatterers, and increased detectability for regions of varying structure. A signal-to-noise analysis was used to show quantitatively how properties of the imaging system influence low-contrast detectability in quantitative ultrasound images. In one experiment, signal-to-noise measurements using phantoms were compared with B-mode imaging for several transducer bandwidths to observe variations in image contrast and speckle noise. The findings are being used to optimise the design of quantitative imaging systems for specific diagnostic tasks.
\end{abstract}

\section{Introduction}

Quantitative methods for characterising biological tissues using ultrasound have been actively pursued for more than two decades. The goal is to enhance the detectability of disease and provide more specific information without diminishing the advantages of current ultrasonographic methods. Those advantages include real-time imaging with relatively little biological risk and low cost.

Many of the approaches to this problem involve measurements of a single acoustic property. For the most part, these approaches have found limited applicability, since no single feature is able to provide sufficient diagnostic information about the diverse range of diagnostic tasks found clinically. Our approach has been to search for the smallest multidimensional feature space capable of detecting and grading disease states. However, we have found that it is also important to be able to measure the acoustic properties at a sufficiently high resolution to display the results in an image. The image format preserves the spatial context of the information so important for diagnosis and is generally accepted by the medical community. Of course, estimates of acoustic properties are limited by their stochastic nature. Precise measurements often require large data samples, resulting in quantitative images with poor spatial resolution. As the spatial resolution increases, so does the uncertainty in the measured parameter which increases image noise and decreases contrast.

This paper focuses on a method for imaging individual properties of soft tissue microstructure using ultrasound. The average scattering particle size and the net

† Originally presented at VIII European workshop on ultrasonic tissue characterisation and echographic imaging (Nijmegen, The Netherlands), 15-18 October 1989. 
scattering strength are the features imaged. These imaging tools can improve lowcontrast detectability and offer new information about the alterations in tissue architecture that accompany many disease conditions of the liver, spleen and kidney. We will show that it is possible to increase contrast and reduce noise by imaging these two features directly, without adversely affecting the spatial resolution. The result is a net increase in low-contrast detectability over conventional ultrasonography. Detectability was measured using the signal-to-noise ratio (SNR) analysis derived by Wagner (Wagner et al 1983, Smith et al 1983) and phantoms containing disk-shaped targets.

\section{Analysis}

\subsection{A model for the interaction between biological tissues and ultrasound}

The attenuation of acoustic energy in a soft tissue medium results from interactions broadly classified as scattering and absorption. Scattering is an elastic process resulting in a change of amplitude, frequency, phase, or direction of propagation of a wave as a consequence of a spatial or temporal non-uniformity in the propagating medium (Chivers 1977). Absorption is an inelastic process by which some portion of the acoustic wave energy is irreversibly converted into internal energy of the propagating medium structure. The sum of scattering and absorptive losses defines the acoustic attenuation. For most soft tissues scattering represents only a small fraction of the total attenuated energy.

Acoustic scattering will occur in media wherever the local density $(\rho)$ and compressibility $(\kappa)$ values deviate from the average values $\left(\rho_{0}, \kappa_{0},\right)$. Acoustic wave propagation in such a non-uniform medium can be described by a wave equation with source functions due to local fluctuations in density and compressibility. The equation for an acoustic pressure wave in a fluid medium containing scatters is (Morse and Ingard 1968)

$$
\boldsymbol{\nabla}^{2} p-\frac{1}{c_{0}^{2}} \frac{\partial^{2} p}{\partial t^{2}}=\frac{1}{c_{0}^{2}} \frac{\partial^{2} p}{\partial t^{2}} \gamma_{\kappa}(\boldsymbol{r})+\boldsymbol{\nabla} \cdot\left(\gamma_{\rho}(\boldsymbol{r}) \boldsymbol{\nabla} \boldsymbol{p}\right)
$$

where

$$
\begin{aligned}
& \gamma_{\kappa}(\boldsymbol{r})=\frac{\kappa(\boldsymbol{r})-\kappa_{0}}{\kappa_{0}}=\text { fractional variation in medium compressibility, } \\
& \gamma_{\rho}(\boldsymbol{r})=\frac{\rho(\boldsymbol{r})-\rho_{0}}{\rho(\boldsymbol{r})}=\text { fractional variation in medium density, }
\end{aligned}
$$

$p=p(\boldsymbol{r}, t)$ is the pressure, $c_{0}$ is the average speed of sound in the medium, $r$ is the position vector, and $t$ is time.

Solutions for the backscattered pressure are well known for pulse-echo imaging conditions (Morse and Ingard 1968, Lizzi et al 1983, Campbell and Waag 1983) and are briefly reviewed below. In formulating a solution we assume that the medium contains only weakly scattering particles, i.e. the Born approximation where $p=p_{\text {incident }}$ $+p_{\text {scattered }}$, such that incident pressure $p_{\text {i }}$ may be substituted for the total pressure $p$ in the wave equation. We also assume the incident pressure is of the time harmonic form $\exp (-\mathrm{i} \omega t)$, where $\omega$ is the angular frequency $2 \pi f$. The backscattered pressure at $\omega$ is given by (Morse and Ingard 1968)

$$
p_{\omega s}(\boldsymbol{r}, t)=\int_{V}\left\{k^{2} \gamma_{\kappa}\left(\boldsymbol{r}^{\prime}\right) p_{\omega \mathrm{i}}\left(\boldsymbol{r}^{\prime} t\right) G_{\omega}\left(\boldsymbol{r}, \boldsymbol{r}^{\prime}\right)+\gamma_{\rho}\left(\boldsymbol{r}^{\prime}\right) \boldsymbol{\nabla}^{\prime} p_{\omega \mathrm{i}}\left(\boldsymbol{r}^{\prime}, t\right) \cdot \boldsymbol{\nabla}^{\prime} G_{\omega}\left(\boldsymbol{r}, \boldsymbol{r}^{\prime}\right)\right\} \mathrm{d}^{3} r^{\prime}
$$


where the Green function is $G_{\omega}\left(\boldsymbol{r}, \boldsymbol{r}^{\prime}\right)=\exp \left(\mathrm{i} k\left|\boldsymbol{r}-\boldsymbol{r}^{\prime}\right|\right) /\left(4 \pi\left|\boldsymbol{r}-\boldsymbol{r}^{\prime}\right|\right),\left|\boldsymbol{r}-\boldsymbol{r}^{\prime}\right|$ is the distance between the transducer and the scattering volume $V$, and $k$ is the wave number $=\omega / c$.

The incident pressure field from a single-element focused transducer, typical of pulse echo imaging, is described by the expression

$$
p_{\omega \mathrm{i}}(\boldsymbol{r}, t)=\mathrm{i} \rho_{0} c_{0} k A(\boldsymbol{r}, k) U(\omega) \exp (-\mathrm{i} \omega t)
$$

where

$$
A(\boldsymbol{r}, k)=\frac{1}{2 \pi} \int_{A_{0}} \frac{\exp \left(\mathrm{i} k\left|\boldsymbol{r}-\boldsymbol{r}^{\prime}\right|\right)}{\left|\boldsymbol{r}-\boldsymbol{r}^{\prime}\right|} \mathrm{d} \boldsymbol{A}_{0} .
$$

The transducer surface is assumed to move uniformly with speed $U(\omega) \exp (-i \omega t)$ and the radiating surface area of the transducer is $A_{0}$. Substituting the expression for $p_{\omega i}$ in equations (3) and (4) into (2), and noting that the frequency dependence of compressibility and density source terms are equal for all practical measurement conditions, we can express the force, $f$, on the transducer due to the backscattered pressure as

$$
f_{\omega}(t)=\frac{1}{2} \mathrm{i} \rho_{0} c_{0} k^{3} U(\omega) \exp (-\mathrm{i} \omega t) \int_{V} A^{2}\left(\boldsymbol{r}^{\prime}, k\right) \gamma\left(\boldsymbol{r}^{\prime}\right) \mathrm{d}^{3} r^{\prime}
$$

The force at frequency $\omega$ is proportional to the relative variation in the acoustic impedance of the particle from the average value, $\gamma(\boldsymbol{r})=\gamma_{\kappa}(\boldsymbol{r})-\gamma_{\rho}(\boldsymbol{r})$, weighted by the square of the incident field $A(r, k)$, and integrated over the entire scattering volume $V$. The quantity $A(r, k)$ is squared because the same transducer is used to both transmit and receive pressure waves.

The echo signal $s_{\mathrm{m}}$ measured in an experiment is expressed by multiplying equation (5) by the acousto-electric transfer function $T(\omega)$ and integrating over all frequencies.

$$
s_{\mathrm{m}}(t)=\int_{-\infty}^{\infty} T(\omega) f_{\omega}(t) \mathrm{d} \omega .
$$

The echo signal is localised spatially by multiplying $s_{\mathrm{m}}$ by a gating function $g(\boldsymbol{r})$. Fourier transforming the result yields the echo signal spectrum $S_{\mathrm{m}}$ which may be expressed as a function of the spatial frequency variable $k$.

$$
S_{\mathrm{m}}(k)=\mathrm{i} k^{3} C(k) \int_{-\infty}^{\infty} A^{2}\left(\boldsymbol{r}^{\prime}, k\right) g\left(\boldsymbol{r}^{\prime}\right) \gamma\left(\boldsymbol{r}^{\prime}\right) \mathrm{d}^{3} r^{\prime} .
$$

The factor $C(k)=\frac{1}{2} \rho_{0} c_{0} T(\omega) U(\omega)$ describes frequency-dependent instrument effects, namely, the pulse spectrum and the acousto-electric transfer function. This factor can be eliminated by dividing $S_{\mathrm{m}}$ by the echo signal spectrum from a reference target, for example, a planar reflecting surface. Spectral normalisation is a standard procedure in acoustic measurements for removing instrument effects and obtaining spectral estimates that are representative of the scattering medium (Campbell and Waag 1983, Lizzi et al 1983). Using a technique described previously (Insana et al 1990) and assuming all measurements are confined to a region near the transducer focus, the normalised echo signal spectrum $S$ is given by

$$
S(k)=\frac{\mathrm{i} A_{0} k^{3}}{\left(2 \pi R_{1}\right)^{2}} \int_{-\infty}^{\infty} H^{2}\left(x_{0}, y_{0}\right) g\left(z_{0}\right) \gamma\left(r_{0}\right) \exp \left(\mathrm{i} 2 k r_{0}\right) \mathrm{d}^{3} r_{0} .
$$

$H^{2}\left(x_{0}, y_{0}\right)$ is the transducer-beam pulse-echo directivity function in a plane normal to the beam axis, and $g\left(z_{0}\right)$ is the range gate (figure 1 ). The product of these two functions 


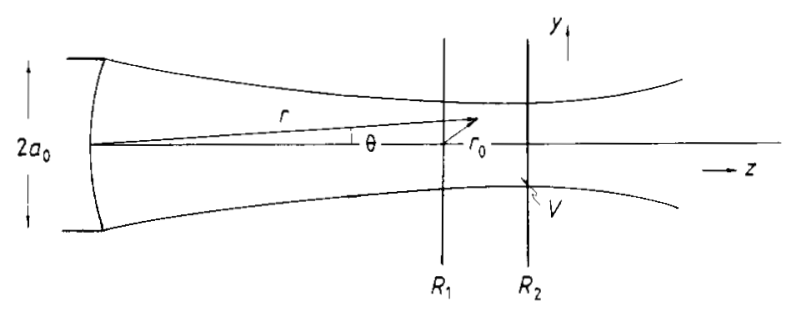

Figure 1. Transducer geometry in the $y, z$ plane. The diameter of the transducer is $2 a_{0}$, the scattering volume is $V$, and the range gate is bounded by $R_{1}$ and $R_{2}$.

defines the distribution of acoustic energy in the scattering volume. The function $\gamma\left(\boldsymbol{r}_{0}\right)$ describes the geometry and relative impedence of the scatterers, and the exponential factor is the Fourier kernel.

The normalised backscattered power spectrum $W(k)$ is estimated from the meansquare of the spectrum $S$. For statistically homogeneous media, the averaged spectrum for a region of interest ( $\mathrm{ROI}$ ) is given by

$$
W(k)=\frac{1}{N_{l}} \sum_{l=1}^{N_{i}}\left|S_{l}\left(k, Z_{l}\right)\right|^{2},
$$

where $N_{l}$ is the number of gated waveform segments of length $Z_{l}$. We have assumed that biological soft tissues are weakly stationary, which means the expected signal intensity is constant over the analysis volume and the autocorrelation function depends on the relative position, $\Delta r$ rather than the absolute position. Consequently, the data are ergotic; that is, temporal averaging is equivalent to ensemble averaging. Combining equations (8) and (9) yields an expression for the backscattered power spectrum:

$$
W(k)=\frac{A_{0}^{2} k^{6}}{\left(2 \pi R_{1}\right)^{4}} \int_{-\infty}^{\infty} B_{H}(\Delta x, \Delta y) B_{g}(\Delta z) B_{\gamma}(\Delta r) \exp (\mathrm{i} 2 k \Delta r) \mathrm{d}^{3} \Delta r,
$$

where $B_{H}, B_{g}$ and $B_{\gamma}$, are the autocorrelation functions for the transducer-beam directivity, the range gate, and the scattering medium, respectively. In most tissue parenchyma, the correlation langths for scattering structures are presumed to be much smaller than those for the directivity function and range gate. Therefore $B_{y}$ becomes negligibly small before either $B_{H}$ or $B_{g}$ changes significantly, and we can assume $B_{H}$ and $B_{g}$ are constants given by their value at $\Delta r=0$ (Campbell and Waag 1983). The power spectrum is then approximately equal to

$$
W(k) \simeq \frac{A_{0}^{2} k^{6}}{\left(2 \pi R_{1}\right)^{4}} B_{H}(0,0) B_{g}(0) \int_{-\infty}^{\infty} B_{\gamma}(\Delta r) \exp (\mathrm{i} 2 k \Delta r) \mathrm{d}^{3} \Delta r
$$

where $B_{H}(0,0)$ and $B_{g}(0)$ can easily be calculated for the experimental conditions, e.g. transducer aperture, distance between the transducer and the sample, and the range gate length.

The backscatter coefficient $\sigma$, which is defined as the differential scattering cross section per unit volume at $180^{\circ}$, may be expressed as

$$
\sigma=\frac{k^{4}}{16 \pi^{2}} \int_{-\infty}^{\infty} B_{\gamma}(\Delta r) \exp (\mathrm{i} 2 k \Delta r) \mathrm{d}^{3} \Delta r
$$


In equation (12) we assume the scattering is incoherent, that is, that the pressure waves from individual scatterers do not interfere, but sum incoherently to give the total backscattered intensity. Backscatter coefficients are particularly useful for describing structural properties of the medium, such as the composition and geometry of the scattering sites, from measurements of the echo signal power spectrum. Combining the expression above with equation (11) yields an equation for $\sigma$ in terms of $W$,

$$
\sigma \simeq \frac{1.45 R_{1}^{2}}{A_{0} \Delta z} W(k)
$$

$B_{H}(0,0)$ was computed assuming a spherically focused transducer of area $A_{0}$, and $B_{g}(0)$ was computed assuming a Hanning window of length $\Delta z=R_{2}-R_{1}$ (see figure 1 ), as described by Insana et al (1990).

The frequency dependence of $\sigma$ depends on the scattering particle size, shape and elastic properties, while its magnitude depends on the scatterer size, number density, $\bar{n}$, (number per unit volume) and scattering strength per particle, $\gamma_{0}^{2}$ (squared fractional variation in acoustic impedance between scatterers and surrounding medium). Not all of these properties can be estimated concurrently; some prior knowledge of the medium is required. For example, the autocorrelation function and elastic properties must be known to accurately estimate the scatterer size, number density and scattering strength. Although many of these properties are still poorly defined for soft biological tissues, several research groups have demonstrated relationships between acoustic spectra measured in liver parenchyma and structures seen using optical microscopy. For example, see (Nicholas 1979, Nassiri and Hill 1986, Lizzi et al 1987). In these studies elementary correlation models (e.g. Gaussian functions) were used to probe tissues, and collagen was assumed to be the dominant scattering material.

\subsection{Testing the model}

Backscatter coefficients were measured using equation (13) for two well-defined test media consisting of glass microspheres in agar. These results, as plotted in figure 2,

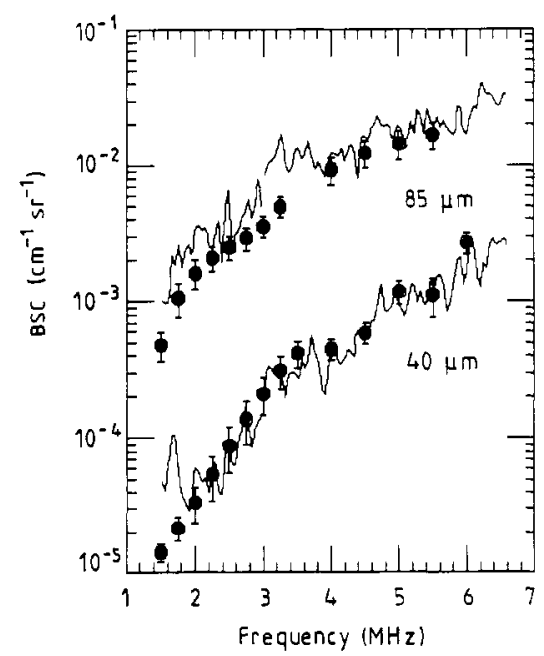

Figure 2. Comparison between BSC measurements using equation (13) (noisy lines) and the technique of Madsen et al (1984) (solid dots). Two different test samples were used: one contains $40 \mu \mathrm{m}$ and the other $85 \mu \mathrm{m}$ diameter glass spheres in agar. Error bars indicate one standard deviation of the mean value. 
were found to agree with those of a more general method for computing backscatter coefficients (Madsen et al 1983). The method of Madsen et al has been shown to agree with acoustic scattering theory. Backscatter coefficient measurements were then used to estimate the diameter $(D)$ and net scattering strength $\left(\bar{n} \gamma_{0}^{2}\right)$ of the spheres. These quantities were determined by selecting the correlation function model that most accurately accounts for the frequency dependence of the backscatter coefficient data. The correlation model depends on the shape and elastic properties of the scatterers, which means that some prior knowledge of the source of scattering is essential for accurate measurements of $D$ and $\bar{n} \gamma_{0}^{2}$. For the test samples, the scatterers were spherical, and the essential elastic properties of the glass are density $\left(2.38 \mathrm{~g} \mathrm{~cm}^{-3}\right)$, longitudinal speed of sound ( $5570 \mathrm{~m} \mathrm{~s}^{-1}$ ) and Poisson ratio (0.21). The surrounding agar is fluid-like (Poisson ratio $\simeq 0.5$ ), the speed of sound is $1545 \mathrm{~m} \mathrm{~s}^{-1}$ and the density is $1.0 \mathrm{~g} \mathrm{~cm}^{-3}$. The appropriate correlation functions are spherical or Gaussian functions (Insana 1990). In soft tissues, the collagenous structures have very complex shapes and poorly defined elastic constants. Although the appropriate correlation models have not been measured, a simple Gaussian model is currently used to size tissue scatterers until a more detailed description is developed from studies involving acoustic microscopy. (For example, see Sherar et al 1987.)

To estimate scattering particle sizes from backscatter coefficient estimates, we first compute a set of correlation model functions for a wide range of possible particle sizes, and store the results in a look-up table. Each modelled function is then compared with the backscatter coefficient data over the frequency bandwidth, and, using a standard least-squares technique, $D$ is estimated by determining which model function has the minimum average squared deviation with the data. The method is most sensitive to particles in the wavelength range $0.5 \lambda \leqslant \pi D \leqslant 1.2 \lambda$. For the 1 to $10 \mathrm{MHz}$ range of frequencies used in diagnostic ultrasound, particle sizes in the range of 20 to $500 \mu \mathrm{m}$ may be measured. The quantity $\bar{n} \gamma_{0}^{2}$ is determined from the magnitude of the backscatter coefficient, extrapolated to zero frequency.

Results using the data in figure 2 are summarised in table 1 . Details of the measurement technique and an extensive list of phantom measurements are described by Insana et al (1990). The following section outlines the application of these methods to quantitative ultrasonic imaging.

Table 1. Comparison of optically and acoustically measured properties of test media.

\begin{tabular}{|c|c|c|c|c|}
\hline \multicolumn{3}{|c|}{ Optically measured values } & \multicolumn{2}{|c|}{ Acoustically estimated } \\
\hline $\begin{array}{l}\text { Sphere } \\
\text { diameter } \\
(\mu \mathrm{m})\end{array}$ & $\begin{array}{l}\text { Number } \\
\text { density } \bar{n} \\
\left(\mathrm{~mm}^{-3}\right)\end{array}$ & $\begin{array}{l}\text { Scattering } \\
\text { strength per } \\
\text { particle } \gamma_{0}^{2}\end{array}$ & $\begin{array}{l}\text { Sphere } \\
\text { diameter } \\
(\mu \mathrm{m})\end{array}$ & $\begin{array}{l}\bar{n} \gamma_{0}^{2} \\
\text { product } \\
\left(\mathrm{mm}^{-3}\right)\end{array}$ \\
\hline $40 \pm 3$ & $14.6 \pm 7.3$ & 2.74 & $40 \pm 7$ & 42 \\
\hline $85 \pm 3$ & $12.1 \pm 6.0$ & 2.74 & $94 \pm 12$ & 23 \\
\hline
\end{tabular}

\section{Imaging applications}

\subsection{Image formation}

In conventional B-scan ultrasonography, the axis of the transducer beam lies in the plane of the image. In C-scan imaging, the beam is perpendicular to the image plane 
$(x, y)$ as shown in figure 3 . One advantage of C-scan imaging is that data outside of the image plane, i.e. along $z$, is used for parameter estimation, thus preserving spatial resolution in the image plane, and allowing the entire image to lie in the focal zone of the transducer. Disadvantages include increased scan times, partial voluming effects, and, for sector scans, a curved scan volume.

With the scanning device shown in figure 3 operated near the transducer's radius of curvature, we obtained a maximum format size of $128 \times 128$, representing an image size of $64 \times 64 \mathrm{~mm}$. One echo signal waveform was recorded for each pixel in the image, at a rate of $25 \mathrm{M}$ samples per second. Range gates, $\Delta z$, between 5 and $15 \mathrm{~mm}$ were used to compute echo spectra and determine backscatter coefficients over the transducer bandwidth, according to equation (13). Subsequently, the two parameters $D$ and $\bar{n} \gamma_{0}^{2}$ were determined, assigned grey level values according to their magnitudes, and mapped into separate grey-scale images. Envelope detected waveforms were also generated for comparison in a manner similar to that used in commercial B-mode imaging systems. All data were reduced off-line and displayed using a VAXstation 3500. Data acquisition and image formation times are currently several minutes per set of three images.

\subsection{Results}

Phantoms were constructed for analysing the imaging properties of the parametric images. Each phantom contains disk-shaped targets in a uniform background. The background and the targets consist of randomly positioned glass microspheres suspended in agar. The distributions of the glass scattering particle diameters are strongly peaked about the mean so that a single particle size could be assumed. The phantoms are cylindrically-shaped, $7.5 \mathrm{~cm}$ in diameter and $2.5 \mathrm{~cm}$ thick. A $1.0 \mathrm{~cm}$ diameter target and a $2.5 \mathrm{~cm}$ diameter target are located in each sample. (Elastic properties of the glass and agar were given in a previous section.)

Examples of one phantom at $5 \mathrm{MHz}$ are displayed in figure 4 . The targets differ from the background by the size and number density of scatterers. In the targets, the average scatterer diameter is $75 \pm 3 \mu \mathrm{m}$ and the number density is $3 \mathrm{~mm}^{-3}$. In the

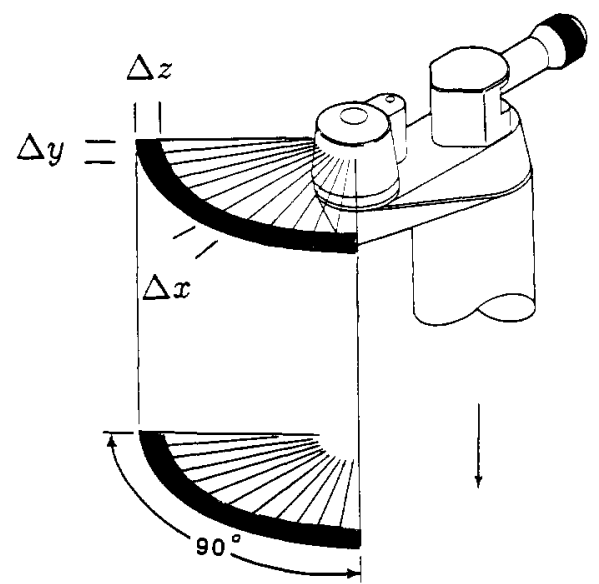

Figure 3. Illustration of C-scan data acquisition. Parameters are estimated from data recorded within the range gate, $\Delta z$ (shaded areas). The horizontal image axis is in the scan plane of the mechanically steered transducer. Distance between waveforms is $\Delta x$. The transducer is translated along the elevation in increments $\Delta y$ to form the vertical axis of the image. 


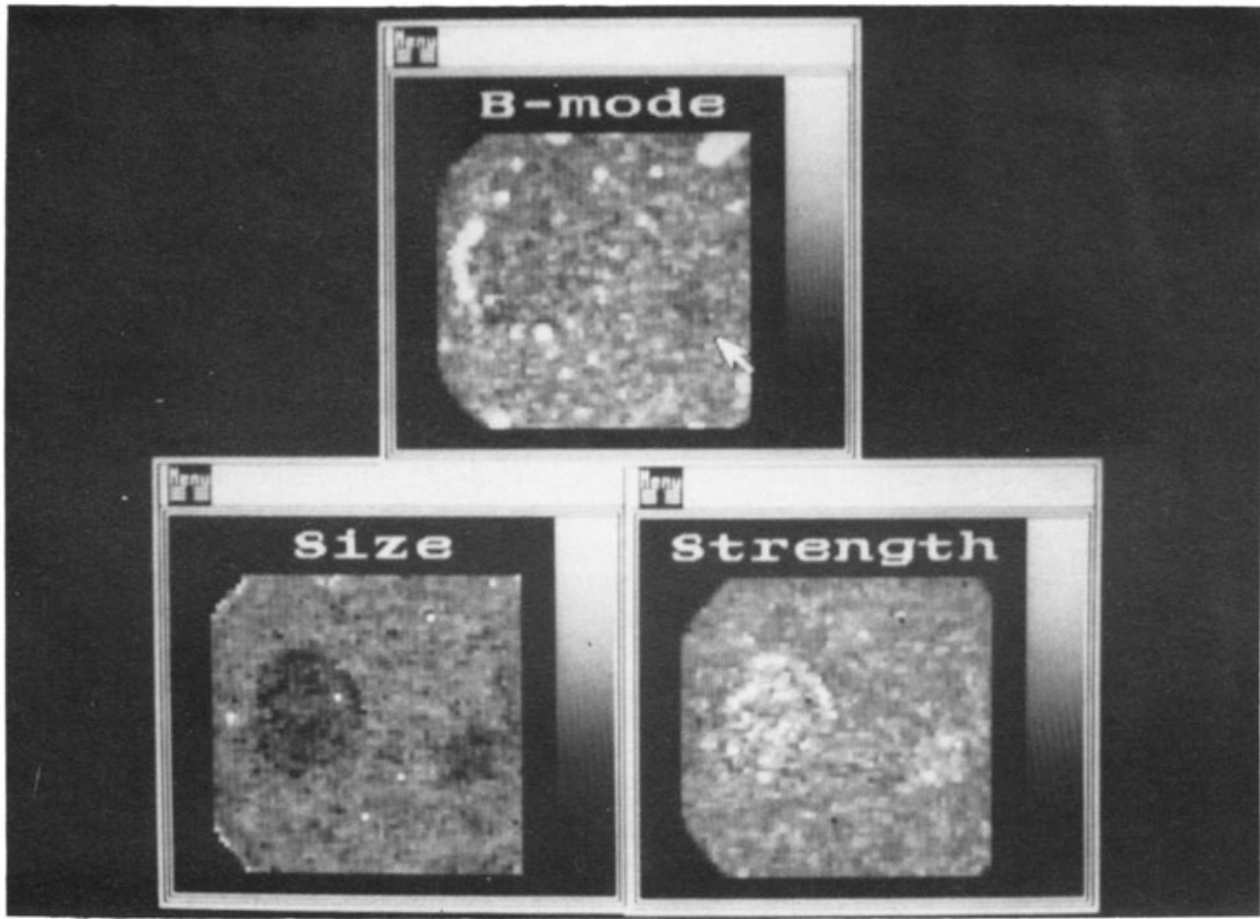

Figure 4. Images of a test sample at $5 \mathrm{MHz}$. On top is a B-mode image of a sample containing a $2.5 \mathrm{~cm}$ low-contrast circular target (left centre) and a $1.0 \mathrm{~cm}$ low-contrast circular target (right centre, indicated by an arrow). On the lower left is the scatterer size image and on the lower right is a scattering strength image calculated from the same data set as the B-mode image.

background, the average scatterer diameter is $105 \pm 4 \mu \mathrm{m}$ and the number density is $1.5 \mathrm{~mm}^{-3}$. In this example, the pixel size is $1 \mathrm{~mm} \times 1 \mathrm{~mm}$.

The top image is a B-mode image, where the pixel values represent the peak echo signal from $20 \mu \mathrm{s}(15 \mathrm{~mm})$ of data. The combined effect of smaller particle size and higher number density results in a lower average brightness for the targets at very low contrast $(0.4 \mathrm{~dB})$. On the lower left is a scatterer size $(D)$ image computed from the same data. Target contrast is increased to $2.4 \mathrm{~dB}$, very close to the object contrast $(2.9 \mathrm{~dB})$, and the $1 \mathrm{~cm}$ diameter target is now clearly visible. In addition, the average pixel values inside and outside the target regions give the acoustic scatterer size estimates of 80 and $106 \mu \mathrm{m}$, respectively. On the lower right is the scattering strength $\left(\bar{n} \gamma_{0}^{2}\right)$ image. The targets appear brighter than the background, corresponding to the higher number density of scatterers inside the targets. (The scattering strength per particle $\gamma_{0}^{2}$ is the same throughout.) The image contrast of $5.6 \mathrm{~dB}$ is very close to the object contrast of $6 \mathrm{~dB}$. In summary, the apparent detectability of the targets in the parametric images was increased over that of the B-mode image, particularly for the $1 \mathrm{~cm}$ target, and the pixel values accurately reflect the structural properties of the media.

\section{Signal-to-noise analysis}

\subsection{Definitions}

We wish to compare the detectability of targets in parametric images with those in B-mode images. The comparison method is well established in signal detection theory; 
it involves determining the signal-to-noise ratio (SNR) for the task of detecting diskshaped targets in correlated (speckle) noise fields. Fortunately, a framework for SNR analysis in ultrasonic imaging has been detailed by Wagner et al (1983) and Smith et al (1983). The specific detection task addressed in this study is to distinguish a uniform low-contrast target of area $S_{A}$ from an average background over a similar area. This task is related to the statistical problem of estimating the difference between the average target signal $X_{1}$ and the average background signal $X_{2} . X_{i}$ can represent any of the parameters imaged, e.g. scatterer size. The 'signal' of interest is $\Delta X=X_{1}-X_{2}$, and the 'noise' is the square root of the sum of variances in the target and background, where $\operatorname{var}(\Delta x)=\left(\operatorname{var}\left(X_{1}\right)+\operatorname{var}\left(X_{2}\right)\right) / M$ and $M$ is the number of independent samples per target area available to the decision maker to test for the presence or absence of the target. The signal-to-noise ratio for detecting low-contrast targets may be expressed as

$$
\begin{aligned}
\operatorname{SNR}_{A} & =\frac{\Delta X}{(\operatorname{var}(\Delta X))^{1 / 2}} \\
& =\frac{\Delta X M^{1 / 2}}{\left(\operatorname{var} X_{1}+\operatorname{var} X_{2}\right)^{1 / 2}} .
\end{aligned}
$$

In the low-contrast limit, $X_{1} \simeq X_{2}=X$. With this assumption, and by multiplying the numerator and denominator of equation (14) by $X$, the $\mathrm{SNR}_{A}$ can be approximated by the expression

$$
\mathrm{SNR}_{A} \simeq \frac{X}{\left(\operatorname{var} X_{1}+\operatorname{var} X_{2}\right)^{1 / 2}} \frac{\Delta X}{X} M^{1 / 2}
$$

The first factor in equation (15) is the point-wise signal-to-noise ratio, $\mathrm{SNR}_{0}$, which describes first-order statistical noise properties of the image. The well-known theoretical value for $\mathrm{SNR}_{0}$ measured for the Rayleigh statistics often found in B-scan imaging does not apply to any of the images used in this study. We determined values for $\mathrm{SNR}_{0}$ directly from the images used in the analysis. The second factor represents the contrast for small signal differences. The third factor, $M^{1 / 2}$, is determined by the number of correlation cell areas $S_{c}$ within the target area $S_{A} . S_{c}$ is an area that is proportional to the square of the correlation cell length $S_{c x}$ in a lateral direction (Wagner 1983).

$$
S_{c x}=\int_{-\infty}^{\infty} \frac{C_{X}(\Delta x)}{C_{X}(0)} \mathrm{d} \Delta x
$$

where $C_{X}(\Delta x)$ is the autocovariance function of the image parameter $X$ along the direction $x$, which is the direction perpendicular to the transducer beam axis. (The beam is assumed to be axially symmetric.) For circular targets of radius $d, M^{1 / 2} \simeq d / S_{c x}$. In equations (14) and (15), we assume the targets are large, such that $S_{A}>S_{c}$.

The axial correlation cell length $S_{c z}$, important in B-scan image analysis, is not explicitly a factor in the above equations for C-scan imaging. However, as shown in the next section, $S_{c z}$ does play an important part in determining the $\mathrm{SNR}_{A}$ for the system. $S_{c z}$ is determined by the pulse duration, and therefore reflects the transducer bandwidth.

\subsection{Applications}

In the course of experimentation, we discovered that the transducer bandwidth was the most influential system parameter determining the appearance of the parametric 
images. This might be expected considering that the total amount of information a system can receive is proportional to the product of the frequency range the system receives (effective bandwidth) and the time the system is available to receive signals (range gate). Often the gate duration used in acquiring data is determined by the application. Therefore, as a first step in our SNR analysis, we decided to measure SNRA for the parametric images as a function of transducer bandwidth, and compare those results to equivalent measurements for B-mode images.

We proceeded by scanning the phantom imaged in figure 4 with a (nominally) $7 \mathrm{MHz}$ broadband transducer. As a rule of thumb, the transducer bandwidth scales with its centre frequency. This high-frequency transducer therefore provided a fairly broad bandwidth of frequencies for analysis. The measured centre frequency was $6.5 \mathrm{MHz}$, and the $6 \mathrm{~dB}$ bandwidth extended symmetrically from $4 \mathrm{MHz}$ to $9 \mathrm{MHz}$.
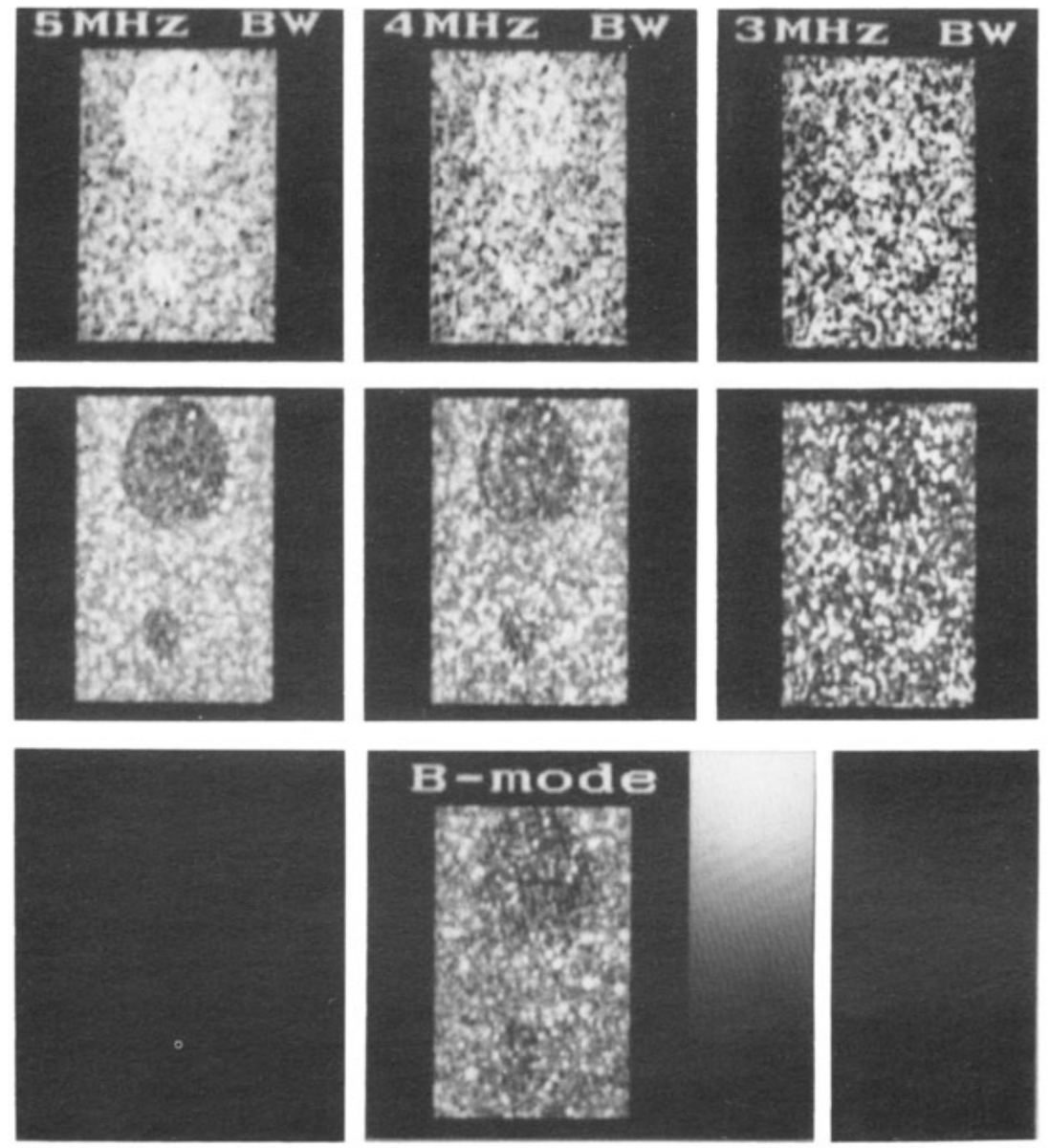

Figure 5. Scatterer size images (top row), scattering strength images (middle row) and a B-mode image (bottom row) of the test sample in figure 4 , measured at $7 \mathrm{MHz}$. The working bandwidth used to estimate parameters varies as indicated at the top. The B-mode image was formed without restricting the bandwidth. The polarity of the targets is reversed relative to figure 4, resulting from measurements made outside the region of sensitivity $(0.5 \leqslant k a \leqslant 1.2)$. Accurate measurements were obtained in the background material. since in the background $k a \sim 1.07$. 
Scatterer size and scattering strength images were formed by selecting a range of frequencies for spectral analysis that extended from the full $5 \mathrm{MHz}$ of bandwidth, down to $3 \mathrm{MHz}$ bandwidth. The reduction in bandwidth was symmetric about the centre frequency. These images, along with the corresponding B-mode image that was formed using the entire bandwidth, are displayed in figure 5 .

The $\mathrm{SNR}_{0}$ and the contrast were determined for each of the three image types as a function of analysis bandwidth. The results for the scatterer size $(D)$ images and the scattering strength $\left(\bar{n} \gamma_{0}^{2}\right)$ images were divided by those for the B-mode image, and plotted in figures 6 and 7. The lateral resolution cell lengths for all three image types were very similar. In addition, $M$ did not change significantly with bandwidth. Using equation (16), $S_{c x}$ was measured for all three image types, and the average found to

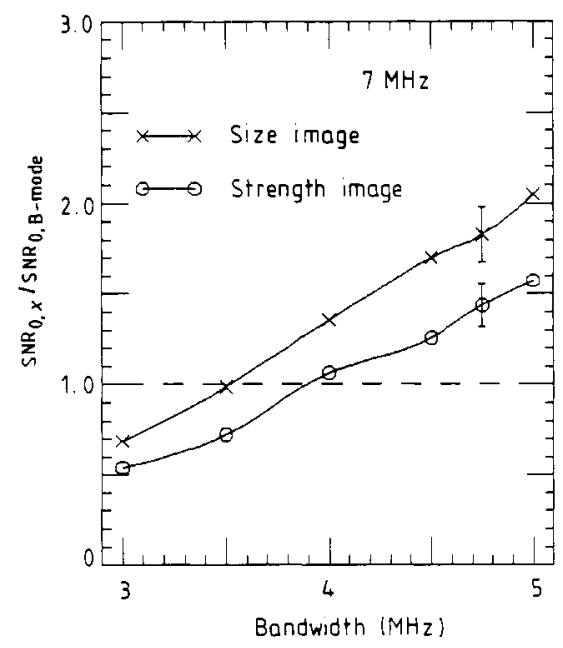

Figure 6. Noise properties measured for the parametric images relative to the B-mode image as a function of bandwidth.

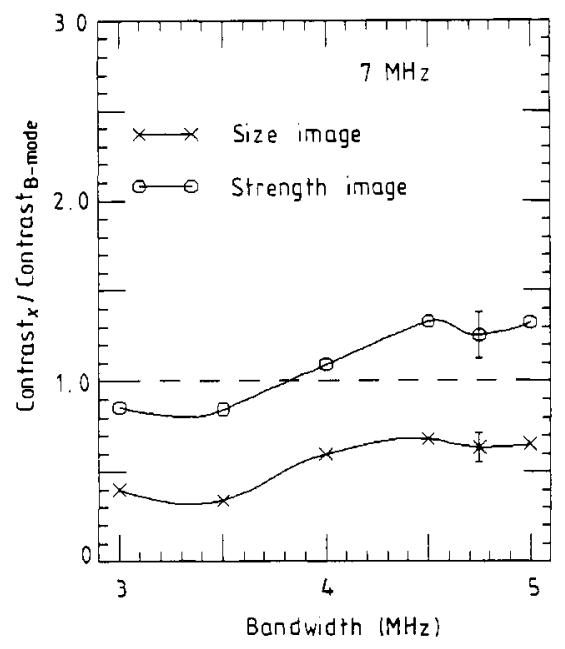

Figure 7. Target contrast measured for the parametric images relative to the B-mode image as a function of bandwidth. 


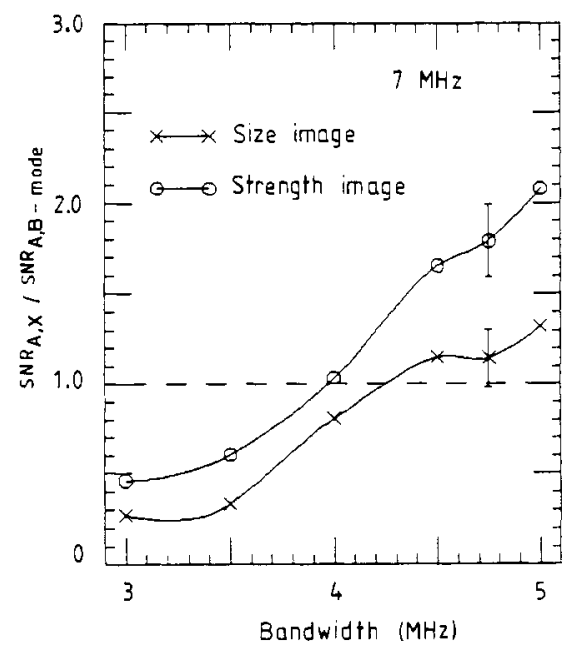

Figure 8. Signal-to-noise ratios for low-contrast target detectability, normalised to that of the B-mode image, is plotted as a function of bandwidth.

be $1.7 \pm 0.3 \mathrm{~mm}$. Therefore, resolution was not a significant factor in the inter-image comparisons for low-contrast detectability.

We computed values of $\mathrm{SNR}_{A}$ for the three image types using equation (15) and the information above. As in figures 6 and 7 , the $\mathrm{SNR}_{A}$ for scatterer size images and scattering strength images were normalised by the corresponding value for the B-mode image. The results are plotted as a function of bandwidth in figure 8 .

\section{Discussion}

The break-even point in $\mathrm{SNR}_{A}$ is at approximately $4.0 \mathrm{MHz}$ bandwidth for scattering strength images and is somewhat higher for scattering size images. This data suggests that percent bandwidths greater than 60 to $70 \%$ are required in order to increase low-contrast detectability using scatterer size and strength images over that of B-mode images.

The only measurable variations in contrast occurred between 3.5 and $4.5 \mathrm{MHz}$, reflecting the limited dynamic range of the task and the breakdown of the correlation model at high frequencies. The contrast in scatterer size images never equalled that of the B-mode image. This finding may be related to the inapplicability of the correlation model used to estimate scatterer sizes at values of $k a>1.2$ ( $k a$ for the $105 \mu \mathrm{m}$ spheres in the background is $\sim 1.5$ at $7 \mathrm{MHz}$ ). The measurements must be repeated for phantoms with smaller-diameter scatterers to determine if these results indicate general trends.

Noise properties of the parametric images $\left(\mathrm{SNR}_{0}\right)$, however, improved linearly with bandwidth over the entire range tested. In this example, improvements in detectability, as measured by $\mathrm{SNR}_{A}$, may be attributed primarily to a reduction in image noise.

Spatial resolution was not a factor in this study, because data were acquired in a C-scan plane. To obtain the high frame rates necessary for diagnostic imaging, data will have to be acquired in the B-scan image plane, where all the analysis data are in the plane of the image. Consequently, contrast and noise in parametric images will greatly depend on the amount of data used to estimate the parameters-the spatial 
resolution. We expect the results of figure 8 will change significantly when imaging in the B-scan plane.

In summary, improved low-contrast detectability has been demonstrated with quantitative ultrasonic imaging methods. The improvements are due to the ability of the technique to separate the contributions of scatterer size and scattering strength to the backscatter signal. The measurement accuracy observed greatly depended on the centre frequency and bandwidth of the interrogating pulse, as do image contrast and noise properties. Acquiring data in a $\mathrm{C}$-scan image plane allowed us to form quantitative images at the limit of resolution determined by the imaging system. The concomitant penalties include longer data acquisition times, larger slice thicknesses, and, for sector images, curved image planes. In all, quantitative ultrasound imaging is a promising new technique for obtaining a more direct interpretation of diagnostic ultrasound image data and increasing low-contrast detectability.

\title{
Acknowledgment
}

This work was supported in part by grants from the Whitaker Foundation, the West Fund and the National Institutes of Health, BRSG S07RR05373.

\begin{abstract}
Résumé
Caractérisation des microstructures d'un milieu aléatoire à l'aide des ultrasons.

Ce travail décrit une technique de formation à l'aide d'ultrasons correspond à des diffuseurs de taille et de pouvoir diffusant moyens, répartis dans un milieu aléatoire. Les images ultrasonores quantitatives permettent une interprétation plus directe des structures internes du milieu, telles que par exemple la taille, la forme, le nombre et les propriétés élastiques des diffuseurs; elles permettent également d'améliorer la détectabilité pour des régions de structure variable. Les auteurs ont utilisé l'analyse du rapport signal sur bruit pour montrer quantitativement comment les propriétés d'un systéme d'imagerie influencent la détectabilité à bas contraste dans les images ultrasonores quantitatives. Ils ont comparé, lors d'une expérimentation, les mesures du rapport signal sur bruit dans des fantômes à l'imagerie en mode B avec diverses bandes passantes de transducteurs pour observer les variations du contraste de l'image et du bruit. Les résultats sont utilisés pour optimiser la conception de systèmes d'imagerie quantitative en fonction d'applications spécifiques du radiodiagnostic.
\end{abstract}

\section{Zusammenfassung}

Charakterisierung der Mikrostrukturen beliebiger Medien mit Hilfe von Ultraschall.

Die vorliegende Arbeit beschreibt ein Ultraschall-Verfahren zur Darstellung von Bildern der durchschnittlichen Größe und des Streuvermögens von Streusubstanzen in einem beliebigen Medium. Quantitative Ultraschalbilder liefern eine direktere Interpretation der zugrundeliegenden Strukturen des Mediums, z.B. von Größe, Form, Anzahl und von den eleastischen Eigenschaften der Streusubstanzen und weisen eine höhere Nachweisbarkeit auf für Gebiete unterschiedlicher Strukturen. Eine Analyse des Signal-RauschVerhaltens wurde verwendet, um quantitativ zu zeigen wie die Eigenschaften des bildgebender Systems die Nachweisbarkeit von Strukturen niedriger Kontraste bei quantitativen Ultraschallbildern beeinflussen. In einem Experiment wurden Messungen des Signal-Rausch-Verhältnisses mit Hilfe von Phantomen verglichen mit B-Mode-Bildern verschiedener Wandlerbandbreiten, um so Schwankungen des Bildkontrastes und -Rauschens zu beobachten. Die Ergebnisse werden verwendet zur Optimierung des Aufbaus quanititativer Abbildungssysteme für spezielle diagnostische Aufgaben.

\section{References}

Campbell J A and Waag R C 1983 Normalization of ultrasonic scattering measurements to obtain average differential scattering cross sections for tissues J. Acoust. Soc. Am. 74 393-9 
Chivers R C 1977 The scattering of ultrasound by human tissues-some theoretical models Ultrasound Med. Biol. 3 1-13

Faran J J 1951 Sound scattering by solid cylinders and spheres J. Acoust. Soc. Am. 23 405-18

Insana M F, Wagner R F, Brown D G and Hall T J 1990 Describing small-scale structure in random media using pulse-echo ultrasound $J$. Acoust. Sac. Am. 87 179-92

Lizzi F L, Greenbaum M, Feleppa E J and Elbaum M 1983 Theoretical framework for spectrum analysis in ultrasound tissue characterization $J$. Acoust. Soc. Am. 73 1366-71

Lizzi F L, Ostromogilsky M, Feleppa E J, Rorke M C and Yaremko M M 1987 Relationship of ultrasonic spectral parameters to features of tissue microstructure IEEE Trans. Ultrason. Ferroelec. Freq. Contr. UFFC-34 319-29

Madsen E L, Insana M F and Zagzebski J A 1984 Method of data reduction for accurate determination of acoustic backscatter coefficients J. Acoust. Soc. Am. 76 913-23

Morse P M and Ingard K U 1968 Theoretical Acoustics (New York: McGraw-Hill) ch 8

Nassiri D K and Hill C R 1986 The use of angular scattering measurements to estimate structural parameters of human and animal tissues J. Acoust. Soc. Am. 79 2048-54

Nicholas D 1979 Evaluation of backscattering coefficients for excised human tissues: results interpretation and associated measurements Lltrasound Med. Biol. 8 17-22

Sherar M D, Noss M B and Foster F S 1987 Ultrasound backscatter microscopy images the internal structure of living tumour spheroids Nature 33 493-5

Smith S W, Wagner R F, Sandrik J M and Lopez H 1983 Low contrast detectability and contrast/detail analysis in medical ultrasound IEEE Trans. Son. Ultrason. SU-30 164-73

Wagner R F, Smith S W, Sandrik J M and Lopez H 1983 Statistics of speckle in ultrasound B-scans IEEE Trans. Son. Ultrason. SU-30 156-63 